\title{
Computing the rate of spread of linear flame fronts by thermal image processing
}

\author{
E. Pastora , A. Àgueda ${ }^{\mathrm{a}}$, J. Andrade-Cetto ${ }^{\mathrm{b}}$, M. Muñoz ${ }^{\mathrm{a}}$, Y. Pérez $^{\mathrm{a}}$, E. Planasa,** \\ ${ }^{a}$ Centre for Studies on Technological Risk (CERTEC), Universitat Politècnica de Catalunya, ETSEIB, \\ Diagonal 647, Pav.G, Planta 2, 08028-Barcelona, Catalonia, Spain \\ ${ }^{b}$ Centre de Visió per Computador (CVC), Universitat Autònoma de Barcelona \\ Edifici O, Bellaterra 08193, Spain
}

\begin{abstract}
This work proposes a new thermal image processing method for computing the rate of spread (ROS) of forest fires. It is based on an application for linear flame fronts that are generated on flat surfaces with known dimensions. In the first step of the method, the correspondence between the points of the thermal image obtained and the real plane is calculated by means of a direct linear transformation (DLT). Subsequently, the position of the flame front is determined by applying a threshold-valuesearching criterion within the temperature matrix of the target surface. The design principles for the implementation of this method in the laboratory are described in depth, as well as the considerations that must be taken into account if the method is to be correctly extrapolated to more complex experimental scenarios, such as prescribed burnings or real forest fire emergencies.
\end{abstract}

Keywords: forest fire, homography, thermography, rate of spread

*Corresponding author. Centre for Studies on Technological Risk (CERTEC), Universitat Politècnica de Catalunya.

Tel.: +34-93-401-17-36; fax: +34-93-401-71-50.

E-mail address: eulalia.planas@upc.edu (E. Planas). 


\section{Introduction}

Rate of spread (ROS) is one of the most significant parameters in the description of forest fire behaviour, since it is directly related to fire intensity [1] and flame front geometry [2] and, thus, to the levels of danger associated with its propagation. In forest fire emergency management, knowing this parameter is crucial. Therefore, a great deal of scientific research is devoted to this field, which centres either on mathematical modelling [3] or on experimental assessment for the validation of models.

Various methods can be used to experimentally assess the ROS, and their success depends on the extent to which straightforward implementation, reliability, precision, feasibility of extrapolation to other experimental scenarios (small scale, field scale and real scale) and cost come together.

Historically, very simple conceptual procedures have been used to experimentally determine the ROS. These are based on calculating the time that the front takes to cover certain known distances, by means of the integration of several warning systems within the experimental scenario. Thus, a known linear arrangement of warning elements of an acoustic, mechanical, electrical or simply visual nature, which go off and give a signal during the passage of a fire front, makes it easy to calculate the ROS immediately. One of the most commonly used systems consists in the equidistant positioning of cotton or nylon threads perpendicular to the fire spread direction, in such a way that when the fire spreads it burns through them along its path. By recording the moment at which each thread breaks and by knowing its position, the rate of spread is calculated as the derivative of the distance in relation to time. This system is applicable to experimental fires on a laboratory scale, but it is difficult to extrapolate to field experiments, as it is restricted to those areas in which the features of the landscape and the fuel cover allow the threads to be fixed and observed. It is often difficult to obtain wholly reliable measurements of the ROS because of impaired visibility due to such factors as smoke formation, safety distances and vegetation structure. Moreover, this method only serves to measure the ROS from the most advanced sector of the front, which is the maximum ROS value, since this is the part that breaks the thread and, therefore, determines the point in time to be recorded. Thus, in those cases in which the fire deviates substantially from its linearity, this measurement is not representative of the spreading of the whole fire front. 
In order to overcome some of these drawbacks, a very similar technique is often used in which the fixed parameter is not the position of the marker but the time. This procedure consists in throwing numbered metal sticks into the flame front at known time intervals [4]. Once the fire has passed, the distances between the successive markers are measured in order to match them with the time they were thrown and thus obtain the ROS. Nevertheless, this technique also has shortcomings, such as its dependency on the accuracy of the throwing and on whether the markers are actually found.

These types of methods are not technically complex, nor do they entail very high costs. However, their precision and feasibility decrease drastically when they are extrapolated to more complex experimental scenarios. Therefore, they are considered to be totally unfeasible for real forest fire emergencies.

During recent years, in reply to these weaknesses, new techniques have appeared based on discrete or continuous fire monitoring by image acquisition with either IR or video cameras. These techniques are becoming widespread in the field of forest fires due to their high precision and versatility with regard to experimental scenarios $[5,6]$. They are based on the detection of the fire front position by remote sensing; therefore, they are applicable in the most extreme experimental conditions and even in real forest fires. Moreover, rapid advances in technology have made these equipments less costly and therefore more accessible to widespread use. Nonetheless, its use undoubtedly involves technical complexities at all stages (such as the calibration of the equipment in accordance with the experimental scenario and the final computation of the ROS), which means that these techniques are not often used to compute ROS, or that the results may be difficult to optimize once they have been obtained.

Within this group of technologies, the use of IR thermography is perhaps the most paradigmatic, since its potential is huge and the quality of the data that may be extracted is high. This technique allows the fire front position to be detected even in those situations in which the fire spreads accompanied by thick smoke and is therefore invisible to the human eye. Moreover, it allows valuable information about the fire temperature profile $[7,8]$ and the fire intensity to be extracted. However, this technique is one of the most difficult to implement. 
Therefore, the main objective of this work was to develop a simple and very practical method for computing the ROS of a flame front from a sequence of images recorded by an IR camera. The procedure was initially applied to laboratory forest fires, on a flat fuel bed with known dimensions. In the following sections, all the steps are detailed and the technical criteria and the mathematical tools needed for its practical implementation are provided. Finally, technical guidelines for the correct extrapolation of this method to experimental scenarios on a large scale are provided.

\section{Description of the method}

Methods for determining ROS that are based on forest fire monitoring by cameras involve a preliminary step during which the technical conditions for image acquisition are set and then the computation and processing of the images acquired, in order to reach the final ROS value.

Thus, several considerations must first be taken into account in order to select the correct position and calibration parameters of the equipment. In the case of video cameras, this is not a complex procedure, but in IR thermography, certain variables that have a significant influence on the correct functioning of this technology must be identified.

\subsection{Prior tunings}

Suitable parameters must be selected initially to ensure that the IR equipment works correctly. The following items must be taken into consideration:

* Geometric parameters. The first condition that must be taken into account is the position of the camera relative to the target. This decision is not always easy, above all in the case of mobile targets such as fire fronts. It must be determined whether the target will be recorded in a perpendicular or parallel direction, and in the latter case, whether it is better to record the target as it approaches the sensor or as it moves away from it. This decision is often dictated by environmental conditions, but whatever the case, the effects must be assessed, since the IR radiation captured by the sensors strongly depends on the relative position of the emitter and the receiver sources. However, once the direction is ascertained, the distance between the sensor and the target must be 
considered, as it determines the pixel dimension of the images recorded and the optics of the system.

* Environmental parameters. The ambient temperature and relative humidity must be known in order to work with the IR cameras. These variables, together with the distance between the sensor and the target, are needed to estimate the atmospheric transmissivity, which is a key parameter in determining the radiation from the fire that is absorbed by the atmosphere.

* Thermometric parameters. Some attention must be paid to the spectral range that the IR camera can detect, since in certain experimental scenarios it may condition the sensitivity of the camera and the accuracy of the measurement. Finally, the emissivity value of the target must be taken into account; this parameter must be chosen correctly if true values are to be obtained for temperature and heat power [9].

This stage prior to the method for evaluating the ROS is determined by the features of the experimental scenario and by the thermography equipment available. Moreover, the parameters involved are more or less important depending on the use that is ultimately made of the thermal images recorded. Thus, the adequate selection of environmental and thermometric parameters is essential for an accurate study of forest fire temperature profiles, while the fine-tuning of these parameters is not an indispensable requirement for computing the ROS.

Once the above-mentioned parameters have been set, the conditions related to the image acquisition process, i.e. hardware and software requirements, must be considered. At present, recording sequences of thermal images with the IR cameras available on the market may be computer controlled. The minimum advisable hardware requirements are a Pentium III at $1 \mathrm{GHz}$ with $128 \mathrm{Mb}$, although this depends on the IR device used; concerning software requirements, an IR camera-controlling driver with an image acquisition and communications package is needed. Generally, this tool, together with a specific code for thermal image processing, is provided by the manufacturer.

The method developed for computing the ROS makes use of the ThermaCAM ${ }^{\mathrm{TM}}$ Researcher 2001 software for data acquisition, since that is the code that is available commercially. This code allows thermal images to be easily converted into intelligible information for Matlab ${ }^{\circledR}$, which is one of the most 
commonly used mathematical tools in the field of engineering, due to its versatility and computational capacity; and was used in this study to compute the ROS.

\subsection{Computing the rate of spread}

In general, the first issue one must consider when using the majority of these ROS calculation methods is finding the correspondence between the coordinate system of the image, which is expressed in pixels, and the real coordinate system, which is expressed in metres or centimetres. This correspondence makes it possible to locate the real position of the flame front, since its position in the images is known. Once this issue is solved, it is necessary to detect the flame front's position as a function of time in order to ascertain an ROS value.

The calculation method designed involved two steps. The first was to develop a function to compute a matrix of correspondences between the pixels in the image and their position in the real plane. In the second step, another function was defined to detect the position of the flame front as a function of time. Both functions were developed using Matlab ${ }^{\circledR}$; they were named 'homografia.m' and 'velocitat.m' respectively [10]. Their structure and the items considered during their definition are stated below. Nevertheless, before going into any detail, the most important criteria related to the design of the two functions must be taken into account. These criteria are as follows:

a) The technique used to compute the point correspondence matrix demands that the flame front move forward on a flat surface (whether sloped or not) of known dimensions. Despite this restriction, the technique was selected because of its simplicity. The extrapolation of this technique to more complex scenarios is tackled later in this paper.

b) As has already been mentioned, by using the ThermaCAM ${ }^{\mathrm{TM}}$ Researcher 2001 software, it is possible to store varied data of an image in a Matlab ${ }^{\circledR}$ matrix format file. Since the computational space for images is three times higher than for simple matrices, only the temperature matrix (one of the matrices stored in the Matlab ${ }^{\circledR}$ file) was used to detect the flame front's position. This matrix, which is of the same size as the corresponding image, contains the temperature of the corresponding pixel in each cell. Other matrices in the Matlab ${ }^{\circledR}$ file contain operational data on the camera, such as the exact time at which the image was recorded. 


\section{Calculating the homography matrix}

In general, when flame fronts are filmed, either in a laboratory or in an open field, images cannot be recorded from a completely perpendicular position above the advancing flame front's surface. For this reason, a projective vision of the scene is always recorded. This means that filmed surfaces show a geometrical distortion and, thus, the first step in computing the ROS is to determine the correspondence between the points on the recorded image and the real plane.

To solve this issue, a multiple view projective geometry technique was used, and what is known as the planar homography matrix $H$, was determined. An homography is the biunivocal geometric transformation between two planes or of a plane with itself, which transforms in such a way that every straight line on a plane corresponds to a straight line on the other plane, and a point on the first straight line corresponds to a point on the other straight line. Therefore, the $H$ matrix is the one that relates the pixels of the images to their position on the real surface.

If we begin by studying a flat surface, we only need to know the correspondence between four points in the image and their real position to compute the $H$ matrix. If the dimensions of the real surface on which the flame front advances are known, the four vertices can be selected.

If the reference system used is centred on one of the four vertices, the coordinates of the other vertices will be in accordance with the dimensions of the study area and the point of origin selected. In a two-dimensional Euclidian space $\left(\mathrm{R}^{2}\right)$, and for this case study, the coordinates of these four points can be expressed as shown in Figure 1. Given the fact that the plane on which they are located has a certain perspective, these points are in the two-dimensional projective space $\mathrm{P}^{2}$. When working in this space, points are represented by a 3 x 1 vector of homogeneous coordinates. A vector has homogeneous coordinates when its last component is exactly 1 . Therefore, when writing a vector's coordinates, this last component must be appended.

\section{Figure 1.}

The $H$ matrix is calculated by applying the simple direct linear transformation (DLT) algorithm, which is described in detail in [11]. Given that 
$X_{i}^{\prime}$ is a homogeneous column vector that contains the coordinates of the vertex $i$ (for $i=1, \ldots, 4$ ) of the real surface (values are expressed in meters), and

$X_{i}$ is a column vector that contains the coordinates of the vertex $i$ (for $i=1, \ldots, 4$ ) on the image (values are expressed in pixels),

in accordance with the example shown in Figure 2, vectors $X_{i}^{\prime}$ and $X_{i}$ take the following values:

$$
\begin{aligned}
& X_{1}^{\prime}=\left(\begin{array}{l}
0 \\
1 \\
1
\end{array}\right), X_{2}^{\prime}=\left(\begin{array}{l}
0 \\
0 \\
1
\end{array}\right), X_{3}^{\prime}=\left(\begin{array}{l}
1 \\
0 \\
1
\end{array}\right) \text { and } X_{4}^{\prime}=\left(\begin{array}{l}
1 \\
1 \\
1
\end{array}\right) \\
& X_{1}=\left(\begin{array}{c}
21 \\
186 \\
1
\end{array}\right), X_{2}=\left(\begin{array}{c}
57 \\
72 \\
1
\end{array}\right), X_{3}=\left(\begin{array}{c}
274 \\
78 \\
1
\end{array}\right) \text { and } X_{4}=\left(\begin{array}{c}
310 \\
194 \\
1
\end{array}\right)
\end{aligned}
$$

\section{Figure 2.}

For both sets of points, if they are written in a matrix format, Expressions (1) and (2) may be written:

$$
\begin{array}{r}
X^{\prime}=\left[\begin{array}{llll}
X_{1}^{\prime} & X_{2}^{\prime} & X_{3}^{\prime} & X_{4}^{\prime}
\end{array}\right]=\left[\begin{array}{cccc}
0 & 0 & 1 & 1 \\
1 & 0 & 0 & 1 \\
1 & 1 & 1 & 1
\end{array}\right] \\
X=\left[\begin{array}{llll}
X_{1} & X_{2} & X_{3} & X_{4}
\end{array}\right]=\left[\begin{array}{cccc}
21 & 57 & 274 & 310 \\
186 & 72 & 78 & 194 \\
1 & 1 & 1 & 1
\end{array}\right]
\end{array}
$$

In order to ensure that the resulting homography matrix does not depend on the coordinate system in which the points are expressed, both sets of points are normalized separately before the DLT algorithm is applied to compute $H$. The data normalization method consists in isotropically translating and scaling the coordinates, so as to bring the centroid of the set of all points to the origin and so that the average distance of each point from the origin is equal to $2^{1 / 2}$. 
This normalization procedure transforms matrices $X^{\prime}$ and $X$ into $\hat{X}^{\prime}$ and $\hat{X}$ respectively (see Expressions (5) and (6) for this example). In Figure 3, the xy coordinates of points $X_{i}^{\prime}$ and $\hat{X}_{i}^{\prime}$ of matrices $X^{\prime}$ and $\hat{X}^{\prime}$, and the xy coordinates of points $\hat{X}_{i}$ of matrix $\hat{X}$ are graphically represented. The xy coordinates of points $X_{i}$ are not plotted in this figure because their inclusion would make it difficult to visualize the other points.

$$
\begin{gathered}
\hat{X}^{\prime}=\left[\begin{array}{llll}
\hat{X}_{1}^{\prime} & \hat{X}_{2}^{\prime} & \hat{X}_{3}^{\prime} & \hat{X}_{4}^{\prime}
\end{array}\right]=\left[\begin{array}{cccc}
-1 & -1 & 1 & 1 \\
1 & -1 & -1 & 1 \\
1 & 1 & 1 & 1
\end{array}\right] \\
\hat{X}=\left[\begin{array}{llll}
\hat{X}_{1} & \hat{X}_{2} & \hat{X}_{3} & \hat{X}_{4}
\end{array}\right]=\left[\begin{array}{cccc}
-1.4681 & -1.1024 & 1.1024 & 1.4681 \\
0.54356 & -0.61468 & -0.55372 & 0.62484 \\
1 & 1 & 1 & 1
\end{array}\right]
\end{gathered}
$$

Figure 3 .

Once the normalization is applied, the process of obtaining $\hat{H}$ begins. The notation of $\hat{H}$ is accompanied by a hood to make it clear that it will be necessary to denormalize it afterwards.

The DLT is given by the following expression:

$$
\hat{X}_{i}^{\prime}=\hat{H} \cdot \hat{X}_{i}
$$

This condition must be met by all four vertices previously quoted. Thus, the above expression may be written as follows: 


$$
\left[\begin{array}{llll}
\hat{X}_{1}^{\prime} & \hat{X}_{2}^{\prime} & \hat{X}_{3}^{\prime} & \hat{X}_{4}^{\prime}
\end{array}\right]=\left[\begin{array}{lll}
h_{11} & h_{12} & h_{13} \\
h_{21} & h_{22} & h_{23} \\
h_{31} & h_{32} & h_{33}
\end{array}\right] \cdot\left[\begin{array}{llll}
\hat{X}_{1} & \hat{X}_{2} & \hat{X}_{3} & \hat{X}_{4}
\end{array}\right]
$$

The $X_{i}^{\prime}$ vectors and the vectors resulting from the $\hat{H} \cdot \hat{X}_{i}$ multiplication are not equal since they were defined in homogeneous coordinates. They have the same direction but may differ in magnitude by a non-zero scale factor. Therefore, Expression (7) is not always valid. Nevertheless, by expressing this relation in terms of a cross vector product, a simple, linear solution may be found for $\hat{H}$

$$
\hat{X}_{i}^{\prime} \times \hat{H} \cdot \hat{X}_{i}=0
$$

If the $j$-th row of the $\hat{H}$ matrix is denoted by $h^{\mathbf{j} \mathbf{T}}$ and the fact that $\hat{H}$ is a $3 \times 3$ matrix is taken into account, we get:

$$
\hat{H} \cdot \hat{X}_{i}=\left[\begin{array}{c}
h^{\mathbf{T}} \\
h^{2 \mathbf{T}} \\
h^{3 \mathbf{T}}
\end{array}\right] \cdot \hat{X}_{i}=\left(\begin{array}{l}
h^{1 \mathbf{T}} \cdot \hat{X}_{i} \\
h^{2 \mathbf{T}} \cdot \hat{X}_{i} \\
h^{3 \mathbf{T}} \cdot \hat{X}_{i}
\end{array}\right)
$$

in which the superscript "T" included in the notation of $h^{\mathrm{j} \mathbf{T}}$ indicates that it is a row vector.

Moreover, if we write $\hat{X}_{i}^{\prime}=\left(\begin{array}{c}x_{i}^{\prime} \\ y_{i}^{\prime} \\ w_{i}^{\prime}\end{array}\right)$, the cross product may be given explicitly as:

$$
\hat{X}_{i}{ }^{\prime} \times \hat{H} \cdot \hat{X}_{i}=\left(\begin{array}{l}
y_{i}{ }^{\prime} \cdot h^{3 \mathbf{T}} \cdot \hat{X}_{i}-w_{i}{ }^{\prime} \cdot{ }^{\prime} h^{2 \mathbf{T}} \cdot \hat{X}_{i} \\
w_{i}{ }^{\prime} \cdot h^{\mathbf{T}} \cdot \hat{X}_{i}-x_{i}{ }^{\prime} \cdot h^{3 \mathbf{T}} \cdot \hat{X}_{i} \\
x_{i}{ }^{\prime} \cdot h^{2 \mathbf{T}} \cdot \hat{X}_{i}-y_{i}{ }^{\prime} \cdot h^{\mathbf{1 T}} \cdot \hat{X}_{i}
\end{array}\right)=0
$$


Since $h^{\mathbf{j} \mathbf{T}} \cdot \hat{X}_{i}=\hat{X}_{i}^{T} \cdot h^{j}$ and $h^{j}=\left(h^{\mathbf{j} \mathbf{T}}\right)^{T}$, for $j=1, \ldots, 3$, the previous vector may be written in the form of a set of three equations in the entries of $\hat{H}$.

$$
\left[\begin{array}{ccc}
0^{T} & -w_{i}{ }^{\prime} \cdot \hat{X}_{i}^{T} & y_{i}{ }^{\prime} \cdot \hat{X}_{i}^{T} \\
w_{i}{ }^{\prime} \cdot \hat{X}_{i}^{T} & 0^{T} & x_{i}{ }^{\prime} \cdot \hat{X}_{i}^{T} \\
-y_{i}{ }^{\prime} \cdot \hat{X}_{i}^{T} & x_{i}{ }^{\prime} \cdot \hat{X}_{i}^{T} & 0^{T}
\end{array}\right] \cdot\left(\begin{array}{l}
h^{1} \\
h^{2} \\
h^{3}
\end{array}\right)=0
$$

Expression (12) takes the form $\hat{A}_{i} \cdot h=0$, where $\hat{A}_{i}$ is a $3 \times 9$ matrix and $h$ is a column vector with 9 cells. The vector $h$ is made up of the entries of the $\hat{H}$ matrix:

$$
h=\left(\begin{array}{l}
h^{1} \\
h^{2} \\
h^{3}
\end{array}\right)=\left|\begin{array}{l}
h_{11} \\
h_{12} \\
h_{13} \\
h_{21} \\
h_{22} \\
h_{23} \\
h_{31} \\
h_{32} \\
h_{33}
\end{array}\right|
$$

Matrix $\hat{A}_{i}$ is computed for each of the four vertices, and then a new $12 \times 9$ matrix $\hat{A}$ is created with the entries of all four matrices (see Expression (14)).

$$
\hat{A}=\left[\begin{array}{l}
\hat{A}_{1} \\
\hat{A}_{2} \\
\hat{A}_{3} \\
\hat{A}_{4}
\end{array}\right]
$$

To find the values for the entries in $\hat{H}$, the overdetermined set of equations $\hat{A} \cdot h=0$ must be solved. A least squares solution for this set of equations may be computed by decomposing $\hat{A}$ into singular values. Specifically, if $\hat{A}=U \cdot D \cdot V^{T}$ and $D$ runs diagonally and has positive diagonal 
entries, which are arranged in descending order along the diagonal line, then $h$, the singular vector associated to the smallest singular value of $\hat{\mathbf{A}}$, and is given by the last column of $V$.

As a last step, the matrix $H$ is obtained by denormalizing the matrix $\hat{H}$.

The 'homografia.m' function makes it possible to obtain $H$ automatically, as has been explained in preceding paragraphs, independently of the real surface size and of the thermographic camera position. Nevertheless, if the configuration is different from that described in this work and one wishes to use the same function for the determination of $H$, the $X^{\prime}$ matrix must be changed. In addition, the image file name must be replaced with the one corresponding to a recorded image of the own facility.

This matrix is necessary for calculating the rate of spread of the flame front, as explained in the following paragraph.

\section{Calculating the rate of spread}

The images obtained with the thermographic camera allow us to ascertain the temperature corresponding to each pixel in the image, which at the same time corresponds to a given point on the real surface under study. In order to obtain the temperature distribution on the real surface, the area is considered to be a matrix of points, and each of these points will have a corresponding position. From the inverse of the homography matrix obtained from the 'homografia.m' function, the position in the coordinate system associated to the image can be obtained. The temperature value stored in each one of these positions is what is introduced in the matrix corresponding to the surface under study.

In order to determine the position of the flame front at each point in time, it is necessary to establish a search criterion within the temperature matrix corresponding to the surface under study. To simplify this task, the matrix was normalized according to the following equation:

$$
T_{k}^{\prime}=\frac{\left(T_{k}-T_{\text {min }}\right)}{\left(T_{\text {max }}-T_{\text {min }}\right)}
$$

where

$T_{k}^{\prime} \quad$ is the normalized temperature value 
$T_{k} \quad$ is the temperature of the $k$-th element, to which the normalization is being applied

$T_{\min } \quad$ is the minimum temperature of the matrix corresponding to the surface under study

$T_{\max } \quad$ is the maximum temperature of the matrix corresponding to the surface under study

This normalization makes it possible to obtain a new matrix with values ranging between 0 and 1 .

In the normalized matrix of temperatures, the search for the front position is carried out each column at a time, starting from the last line that physically corresponds to the surface side, which is parallel to the ignition line and burns last (see Points A and D in Figure 1). In this way, errors in the localization of the flame front, which stem from the presence of heat points that do not belong to the flame front, are avoided.

The limit value of the normalized temperature from which it is considered that a given point belongs to the flame front corresponds to the input parameter $m$ and can be fixed by the user. In the subsequent paragraph some practical recommendations on the most adequate values for $m$ are given.

For each point in time, the mean position of the flame front and the most advanced position are determined. In this way, the function can calculate two values for the spread rate: a maximum and a mean value. The first value indicates the evolution of the most advanced part of the flame front at each point in time, which is assumed to be similar to the spread rate obtained from other measurement methods, such as the equidistant threads. The second value gives qualitative information about the linearity of the flame front: if it differs greatly from the maximum value, this means that the initial geometry of the flame front has undergone significant modification.

For the calculation of the mean spread rate, it was necessary to define another input variable, cents, which determines the moment at which the 'velocitat.m' function must finalize the search for the flame front position. The cents value is a limit value defined as the number of points on the flame front that have reached the final position on the surface under study (Line AD in Figure 1). The more irregular the flame front profile, the greater the influence of this parameter on the final value of the spread rate. In these cases, if the cents value is too high, the 'velocitat.m' function can account reiteratively for those 
points on the flame front that have reached their final position some moments before, but which continue to burn in the form of incandescent combustion.

Figure 4 shows an example of the results obtained after the execution of the 'velocitat.m' function. Besides the mean and most advanced position of the flame front as a function of time (Figure 4a), a graph with the evolution of the flame position and the geometry of the flame front profile as a function of time is also included (Figure 4b). Due to the generic nature of the function developed, another input variable was defined, int, which allows the interval of images between each profile on the flame front to be selected.

\section{Figure 4.}

\section{Example}

In order to show the validity of the method developed, the 'homografia.m' and 'velocitat.m' functions were applied to several thermographic image sequences that had been recorded during various tests performed in a combustion table at the Centre for Technological Risk Studies (CERTEC) at the Polytechnic University of Catalonia (UPC). In the following paragraph, the main features of the facility and the tests carried out are described. The values assigned to the input variables are also provided, and special attention is paid to parameter $m$. Finally, the results obtained with the method proposed were compared to the results obtained with the traditional threads method.

\subsection{Description of the installation}

The combustion table used in the experimental setup had $4.8 \mathrm{~m}^{2}$ useful burning surface and was equipped with a thermographic camera, a video camera, several thermocouples, a weighting system with a floating surface $(1 \mathrm{~m} \mathrm{x} 1 \mathrm{~m})$ and a balance. Figures $5 \mathrm{a}$ and b show a plan of the installation with the typical position of the thermographic camera during the tests. The camera was able to record the flame front approaching the sensor, with an inclination angle $\alpha$ in relation to the horizontal working plane and centred in relation to the flame front length. In this position, the radiation reaching the thermographic camera comes, on the one hand, from the radiation emitted by the incandescent fuel bed, which is transmitted by the flames, and, on the other hand, from the emission of the flames. 
In Table 1, the technical characteristics of the thermographic camera are shown, together with several adjustments that were made.

\section{Table 1.}

Concerning the direct relation of some of the values shown in Table 1 to the ROS calculation and the criterion used in the determination of the flame front position, the ROS will be more accurate as the temperature values given by the camera are more precise. Thus, the adjusted parameters selected are consistent. In the experimental conditions presented here, the fuel bed can be considered a black body and the emissivity of the flame front, which is characterized by yellow flames between 15 and $20 \mathrm{~cm}$ wide, can be taken as being near to 0.5 [9]. The difference between these two radiation contributions, the greatest of which is the fuel bed, is shown in the thermographic images because the flame contour is completely diluted (Figure 5c). The emissivity of the whole is nearer to that of the black body than to that of the flames. Thus, a value of 0.9 was assigned to the emissivity of the whole, as this is the most suitable value for obtaining precise temperature values.

Concerning the mean distance between the camera and the flame front $(2.70 \mathrm{~m})$ and the optics of the camera, the pixel size is less than $4 \mathrm{~mm}$, which allows an optimal resolution in obtaining the flame front contour.

Finally, the values for ambient temperature and relative humidity were measured and entered in the ThermaCAM ${ }^{\mathrm{TM}}$ Researcher as input parameters for each test. Using these values, the software automatically calculates the atmospheric transmissivity and corrects the temperature values accordingly.

\section{Figure 5 .}

In Figure 5c, an image recorded during the execution of a test can be seen. The quadrilateral that appears in this image indicates the position of the floating study surface. Finally, Figure $5 \mathrm{~d}$ is a temperature isoline map of the surface under study, after the $H$ homography matrix has been applied to the temperature matrix corresponding to the IR image in Figure 5c.

By way of summary, the image sequences used in this study correspond to tests executed without the presence of wind and slope. The tests consist in burning forest fuel beds by creating a linear flame 
front (Figure 6). In [12], the facility, the experimental method and the diverse tests carried out are described in more detail.

\section{Figure 6.}

\subsection{Calculation considerations}

In this practical case, data were only taken from the position of the flame front within the study area or floating surface (Figure 5b). To avoid calculation errors and to reduce the operation time, the initial and final files, which corresponded to images in which it could clearly be seen that the flame front was still not in the area under study or it had already passed it, were eliminated manually.

In order to execute the 'velocitat.m' function, it was necessary to set a numerical value for the input parameters $m$, int and cents, according to the characteristics of the experimental test.

In the case of parameter $m$, a detailed study was carried out of the differences generated in the results of the ROS when different values are assigned to it. By superimposing $m$ isoline maps on the infrared images, it was verified visually that the values of $m$ that were closest to the front position were between 0.1 and 0.3 . According to Equation (14), a value of 0.3 for $m$ was obtained by considering the maximum and minimum temperatures to be equal to $1000 \mathrm{~K}$ and $300 \mathrm{~K}$ respectively (temperatures observed in the analysis of several sequences used in this study) and the instantaneous temperature for

every element $\left(T_{k}\right.$ ) in the order of $500 \mathrm{~K}$, a value that corresponds to the ignition temperature of wheat straw [13], the fuel used in these tests.

The int parameter took different values depending on the specifications of the experimental test corresponding to the sequence of images analyzed. For tests in which the fire advanced faster (approximately $1 \mathrm{~cm} / \mathrm{s}$ ), a value equal to 10 images between every contour profile front was used, while in tests in which the fire advanced more slowly (approximately $0.3 \mathrm{~cm} / \mathrm{s}$ ), a value equal to 25 images was used.

The cents parameter was set at a value equal to 50 points in all cases. Thus, it was assumed that the computed value of the front mean position was representative of the propagation of the whole of the front, while half of the points had reached their final position on the surface under study. Nevertheless, in cases 
in which the flame front loses its linearity completely, it would be advisable to use a lower value for the cents parameter.

\subsection{Results threads vs. 'velocitat.m'}

The maximum rate of spread obtained using the method described and the one obtained with the traditional method of threads were compared for different image sequences. In general, the values obtained by applying the 'velocitat.m' function are slightly higher than those computed for the threads (Figure 7). Nevertheless, the mean value of the differences obtained using one method or the other is less than $6 \%$, a significantly low error rate that is the result of the different nature of the methods. The cases in which this difference is most significant correspond to tests in which there are no time references for some of the threads, or in those cases in which the front had lost its linearity practically from the start of its course.

\section{Figure 7.}

\section{Extrapolating the method to other scenarios}

The method developed using the functions described in the preceding sections is directly applicable to other laboratory devices for fires, since the data required are easy to obtain in any smallscale experimental scenario. Thus, the homography computation function only needs information on the geometry of the fuel bed located on a plane. As far as the rate of spread computation function is concerned, it was defined in a flexible manner, making it possible to change characteristic input parameters depending on the fire scenario.

Most of the work that lies ahead involves adapting the functions described to experimental fires that take place in real scenarios. In these kinds of situations, if the filming is terrestrial, two problems can generally be found. First of all, human figures appear in the IR images that make the flame front difficult to visualize completely. These figures are usually the silhouettes of fire-fighters advancing towards the fire to control its propagation. This difficulty could be overcome by interpolation methods. In most cases, however, the slope of the terrain is not uniform, which strictly precludes the application of the DLT algorithm, since the terrain is made up of different planes. A reasonable solution could be the discretization of the land in a specific number of planes, and consequently, in the same number of 
homography matrixes. Topographical maps of the surface under study would be required for this latter case in order to fix planar patches to the terrain for the computation of the various homographies, one per patch.

As for thermographically determining factors, ambient temperature, relative humidity, absolute distance between camera and object and emissivity of the radiation source are necessary parameters in any experimental scenario. Under experimental conditions in a laboratory, all these data can easily be obtained, except for emissivity. In order to obtain a suitable value for this parameter, specially in those cases in which the thermography equipment uses a spectral range that is sensitive to the selection of this value and there is an overlapping of the sources of radiation, it is necessary to consider, on the one hand, the specific weight of each of these contributions and, on the other, the thickness of the flame. Whatever the case, if this information is not available, reasonable estimates of the parameters are sufficient to obtain equally valid values for the ROS. In experimental conditions on a field scale, estimating the emissivity is simpler, since for thick, intense flames such as those observed in prescribed burnings or real fires, the emissivity is very near to unity [14]. Nevertheless, the distance at which the thermography equipment is positioned in relation to the flame front can be a limiting factor in these scenarios. As the distance between the object and the camera increases, the atmosphere attenuates the fire radiation and the spatial resolution diminishes (the pixel size increases) [15]. The first effect can be corrected by a transmittance factor. The ThermaCAM ${ }^{\mathrm{TM}}$ Researcher 2001 software has a built-in model that computes it according to atmospheric parameters (temperature and relative humidity). Concerning the spatial resolution, the larger the distance between the object and the camera, the less the number of pixels included into the study area. Therefore, the number of pixels can be too low to suitably represent the evolution of the fire and the computed ROS may have an underestimated value. Nevertheless, since the size of the resulting pixel for a fixed distance depends also on the optics of the camera, this effect can be solved by attaching lenses with an adequate field of view to the equipment.

\section{Conclusions}

A method for the fast and accurate calculation of the ROS by processing infrared images was presented in this paper.

Matlab $^{\circledR}$ was used to develop the necessary functions to implement this method, so that it can be freely used and directly applied to the calculation of the ROS by means of thermography in a laboratory. 
The design criteria for the functions were simple and appropriate; it was demonstrated that a normalized DLT algorithm is an appropriate method for calculating the projection of the plane of the image onto the plane of the area under study. Likewise, the selection criterion of the front position by temperature is valid and robust. The practical application and the comparison with the classical method for determining the ROS in a laboratory through the observation of broken equidistant and parallel nylon threads in the advance of the front were successful.

This method is easily applicable to other experimental scenarios that are more complex than the ones described in this paper, such as prescribed burnings or real forest fire emergencies. The guidelines for correct extrapolation have been discussed and compiled and it remains as future work to implement all of them as an extension to the functions described.

\section{Acknowledgments}

This research is supported by the Spanish Ministry of Education and Science under projects DPI 2004-5414, TIC 2003-09291, and a Juan de la Cierva Postdoctoral Fellowship to JAC. It is also supported by the Department of Universities, Research and the Information Society (DURSI) of the Catalan government, the European Social Fund and the Polytechnic University of Catalonia (UPC).

\section{Nomenclature}

$\hat{A}_{i} \quad$ matrix of equation coefficients contributed from the $i$-th correspondence

$\hat{A} \quad$ matrix of equation coefficients built from the matrix rows $\hat{A}_{i}$ contributed from each correspondence

A, B, C, D vertices of the area of study cents maximum number of points of the flame front that have reached the final position on the surface under study

$D \quad$ diagonal matrix with positive diagonal entries, which are arranged in descending order along the diagonal line

DLT direct lineal transformation 
$h$ column vector containing the elements of the $\hat{H}$ matrix

$h^{j} \quad$ column vector of the $\mathrm{j}$-th row of the $\hat{H}$ matrix

$h^{\mathbf{j T}} \quad j$-th row vector of the $\hat{H}$ matrix

$H \quad$ planar homography matrix

$\hat{H} \quad$ normalized planar homography matrix

$i \quad i$-th vertex of the area of study

int interval of images between each profile on the flame front plot

$j \quad j$-th row of the matrix $\hat{H}$

$k \quad k$-th element of the temperature matrix

$m \quad$ limit value of inclusion in the flame front

$\mathrm{P}^{2} \quad$ two-dimensional Projective space

$\mathrm{R}^{2} \quad$ two-dimensional Euclidian space

ROS rate of spread

$T_{k} \quad$ temperature of the $k$-th element of the temperature matrix

$T_{\min } \quad$ minimum temperature of the temperature matrix

$T_{\max }$ maximum temperature of the temperature matrix

$\hat{T}_{k} \quad$ normalized temperature value of the $k$-th element

$U \quad$ unitary matrix which contains the eigenvalues of $\hat{A} \cdot \hat{A}^{T}$

$V \quad$ unitary matrix which contains the eigenvalues of $\hat{A}^{T} \cdot \hat{A}$

$X_{i}^{\prime} \quad$ homogeneous column vector that contains the coordinates of the vertex $i$ of the real surface

$X_{i} \quad$ column vector that contains the coordinates of the vertex $i$ on the image

$\hat{X}_{i}^{\prime} \quad$ homogeneous column vector that contains normalized coordinates of $X_{i}^{\prime}$ 
$\hat{X}_{i} \quad$ homogeneous column vector that contains normalized coordinates of $X_{i}$

$X^{\prime} \quad$ matrix that contains $X_{i}^{\prime}$ vectors

$X \quad$ matrix that contains $X_{i}$ vectors

$\hat{X}^{\prime} \quad$ normalized $X^{\prime}$ matrix

$\hat{X} \quad$ normalized $X$ matrix

$\alpha \quad$ inclination angle in relation to the horizontal working plane

() denotes a vector

[] denotes a matrix 


\section{References}

[1] Byram GM. Combustion of forest fuels. A forest fire control and use. KP Davis (ed). McGrawHill. New York, 1959.

[2] Anderson HE. Fire spread and flame shape. Fire technology. 1968; 4: 51-58.

[3] Pastor E, Zárate L, Planas E, Arnaldos J. Mathematical models and calculation systems for the study of wildland fire behaviour. Progress in Energy and Combustion Science. 2003; 29(2): 139-153.

[4] Rothermel RC, Rinehart GC. Field procedures for verification and adjustment of fire behaviour predictions. General Technical Report INT-142. U.S. Forest Service, Int. For and Rang. Exp. Stn., Ogden, UT, 1983.

[5] Gomez F, Merino L, Arrue B, Ollero A. Aerial monitoring and measurement of forest fires. Progress in biomedical optics and imaging. 2002; 95-105.

[6] Martínez-de Dios JR, Ollero A, Arrue BC. Sistema fuzzy-wavelet para monitorización visual. Aplicación a los incendios forestales. Actas del X Congreso Español Tecnologías y Lógica Fuzzy (ESTYLF), Sevilla. 2000; 371-378.

[7] Aranda JM, Meléndez J, Briz S, De Castro AJ, López F. Measurements of laboratory forest fires with bi-espectral infrared imaging. Forest Fire Research \& Wildland Fire Safety, Viegas (ed.), Millpress, Rotterdam, 2003.

[8] Morandini F, Simeoni A, Santoni PA, Balbi JH. A model for the spread of fire across a fuel bed incorporating the effects of wind and slope. Combustion Science and Technology. 2005; 177 (7): 13811418.

[9] Pastor E, Rigueiro A, Zárate L, Giménez A, Arnaldos J, Planas E. Experimental methodology for characterizing flame emisivity of small scale forest fires using infrared thermography techniques. Forest Fire Research \& Wildland Fire Safety, Viegas (ed.), Millpress, Rotterdam, 2003.

[10] $\quad$ http://certec.upc.es/articles/forestal/rateofspread.htm. 
[11] Hartley R, Zisserman A. Multiple View Geometry in Computer Vision. 2nd Edition, Cambridge: Cambridge University Press, 2003.

[12] Pastor E. Contribució a l'estudi dels efectes dels retardants en l'extinció d'incendis forestals. Doctor Thesis, Universitat Politècnica de Catalunya, Espanya, 2004.

[13] Grotkjaer T, Dam-Johansen K, Jensen AD, Glarborg P. An experimental study of biomass ignition. Fuel. 2003; 82(7): 825-833.

[14] Sullivan AL, Ellis PF, Knight K. A review of radiant heat flux models used in bushfire applications. International Journal of Wildland Fire. 2003; 12: 101-110.

[15] Aranda JM, Meléndez J, Briz J, De Castro AJ, López F, Hernando C, Guijarro M, Martínez E, Madrigal J. Measurements of laboratory forest fires with bi-spectral infrared imaging. Forest Fire Research \& Wildland Fire Safety, Viegas (ed.), Millpress, Rotterdam, 2003. 


\section{List of Table captions}

Table 1. Prior technical characteristics and adjustments to take into account when working with thermography 


\section{List of Figure captions}

Figure 1. Sketch and dimensions of area of study (real surface marked with a dotted line)

Figure 2. Points' correspondences necessary to compute homography

Figure 3. $x y$ coordinates of $X_{i}^{\prime}$ (circle), $\hat{X}_{i}^{\prime}$ (triangle) and $\hat{X}_{i}$ (cross) points

Figure 4. Graphical results of ‘velocitat.m' function. a. Covered distance vs. Time; b. Front profile evolution (every 24 seconds); the arrow of the figure indicates the direction of propagation.

Figure 5. a. Front view of the table of combustion $\left(\alpha=17.2^{\circ}\right)$; b. Top view of the table of combustion; c. IR image of a labor test; $d$. Temperature isolines plot

Figure 6. Test execution image

Figure 7. ROS threads method vs. ROS 'velocitat.m' 


\section{Tables}

Table 1.

\begin{tabular}{|l|l|}
\hline \multicolumn{2}{|c|}{ Technical specifications of the thermographical camera } \\
\hline Commercial name of the equipment & AGEMA Thermovision 570-Pro (FSI- \\
& FLIR Systems) \\
\hline Temperature measurement range / & $-20^{\circ} \mathrm{C}-1500^{\circ} \mathrm{C} / \pm 2 \%$ \\
Precision & \\
\hline Themal sensitivity & $<0.15^{\circ} \mathrm{C}$ \\
\hline Field of view & $24^{\circ} \times 18^{\circ}$ \\
\hline Detector type & FPA $($ Focal Plane Array) \\
\hline Spectral range & $7.5-13 \mu \mathrm{m}$ \\
\hline Operational temperature & $-15^{\circ} \mathrm{C}-50^{\circ} \mathrm{C}$ \\
\hline Storage temperature & $-40{ }^{\circ} \mathrm{C}-70^{\circ} \mathrm{C}$ \\
\hline & Adjustment parameters \\
\hline Mean distance between the camera & $2.70 \mathrm{~m}$ \\
\hline & \\
and the object & $15^{\circ} \mathrm{C}-20^{\circ} \mathrm{C}$ \\
\hline Temperature & $50-70 \%$ \\
\hline Relative humidity & 0.9 \\
\hline Emissivity &
\end{tabular}




\section{List of Table captions}

Table 1. Prior technical characteristics and adjustments to take into account when working with thermography 


\section{List of Figure captions}

Figure 1. Sketch and dimensions of area of study (real surface marked with a dotted line)

Figure 2. Point correspondences necessary to compute the homography

Figure 3. $x y$ coordinates of $X_{i}^{\prime}$ (circle), $\hat{X}_{i}^{\prime}$ (triangle) and $\hat{X}_{i}$ (cross) points

Figure 4. Graphical results of 'velocitat.m' function. a. Covered distance vs. time; b. Front profile evolution (every 24 seconds)

Figure 5. a. Front view of the table of combustion; b. Top view of the table of combustion; c. IR image of a labor test; $d$. Temperature isolines plot

Figure 6. Test execution image

Figure 7. ROS threads method vs. ROS 'velocitat.m' 


\section{Tables}

Table 1.

Technical specifications of the thermographical camera

Commercial name of the

AGEMA Thermovision 570-Pro

equipment

(FSI-FLIR Systems)

Temperature measurement

$-20^{\circ} \mathrm{C}-1500^{\circ} \mathrm{C} / \pm 2 \%$

range / Precision

Themal sensitivity $\quad<0,15^{\circ} \mathrm{C}$

Field of view

$24^{\circ} \times 18^{\circ}$

Detector type

FPA (Focal Plane Array)

Spectral range

7,5- $13 \mu \mathrm{m}$

Operational temperature

Storage temperature

$-15^{\circ} \mathrm{C}-50^{\circ} \mathrm{C}$

$-40{ }^{\circ} \mathrm{C}-70{ }^{\circ} \mathrm{C}$

Adjustment parameters

Mean distance between the

$2.70 \mathrm{~m}$

camera and the object

Temperature

$15-20{ }^{\circ} \mathrm{C}$

Relative humidity

$50-70 \%$

Emissivity 


\section{Figures}

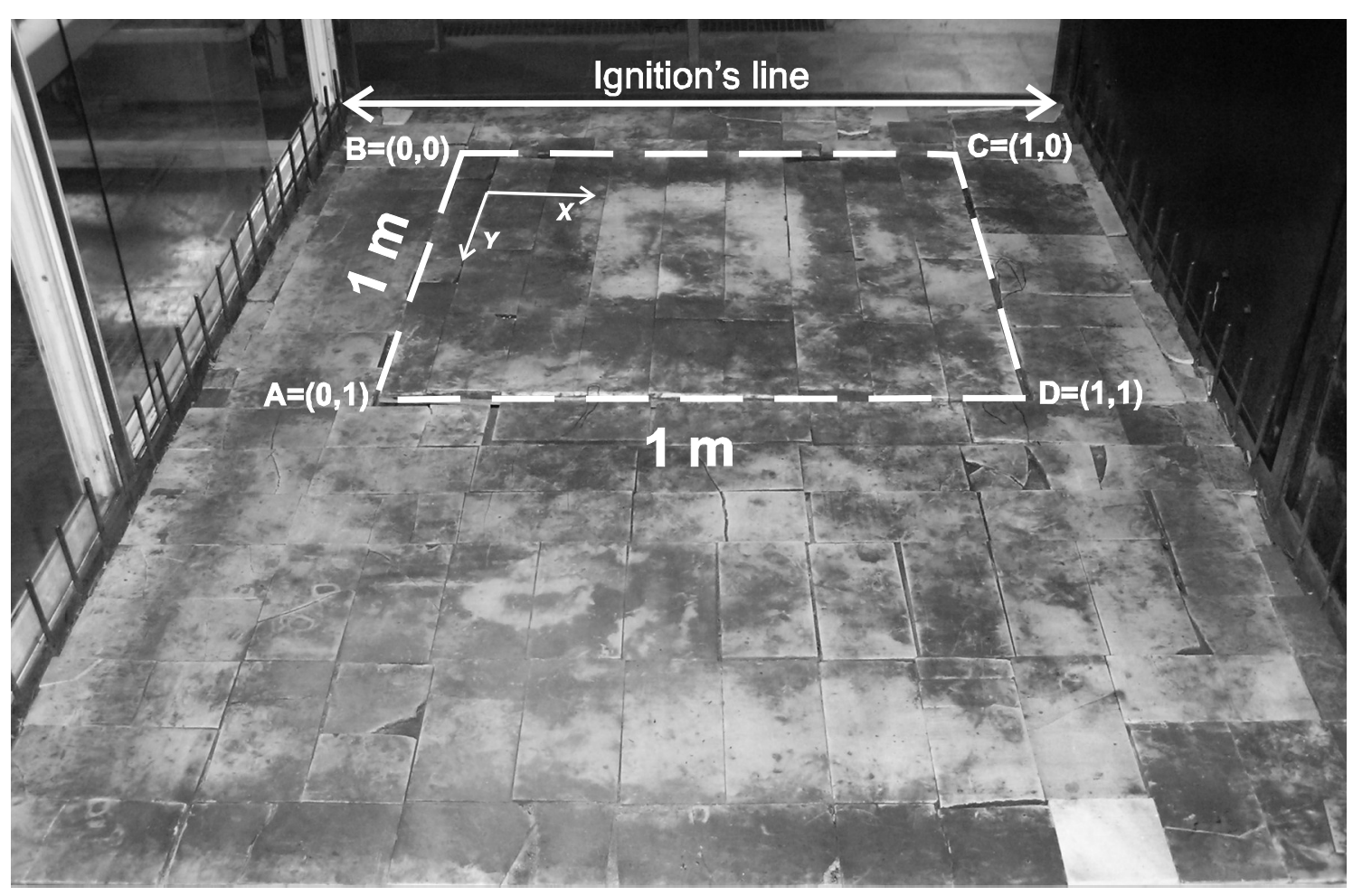

Figure 1.

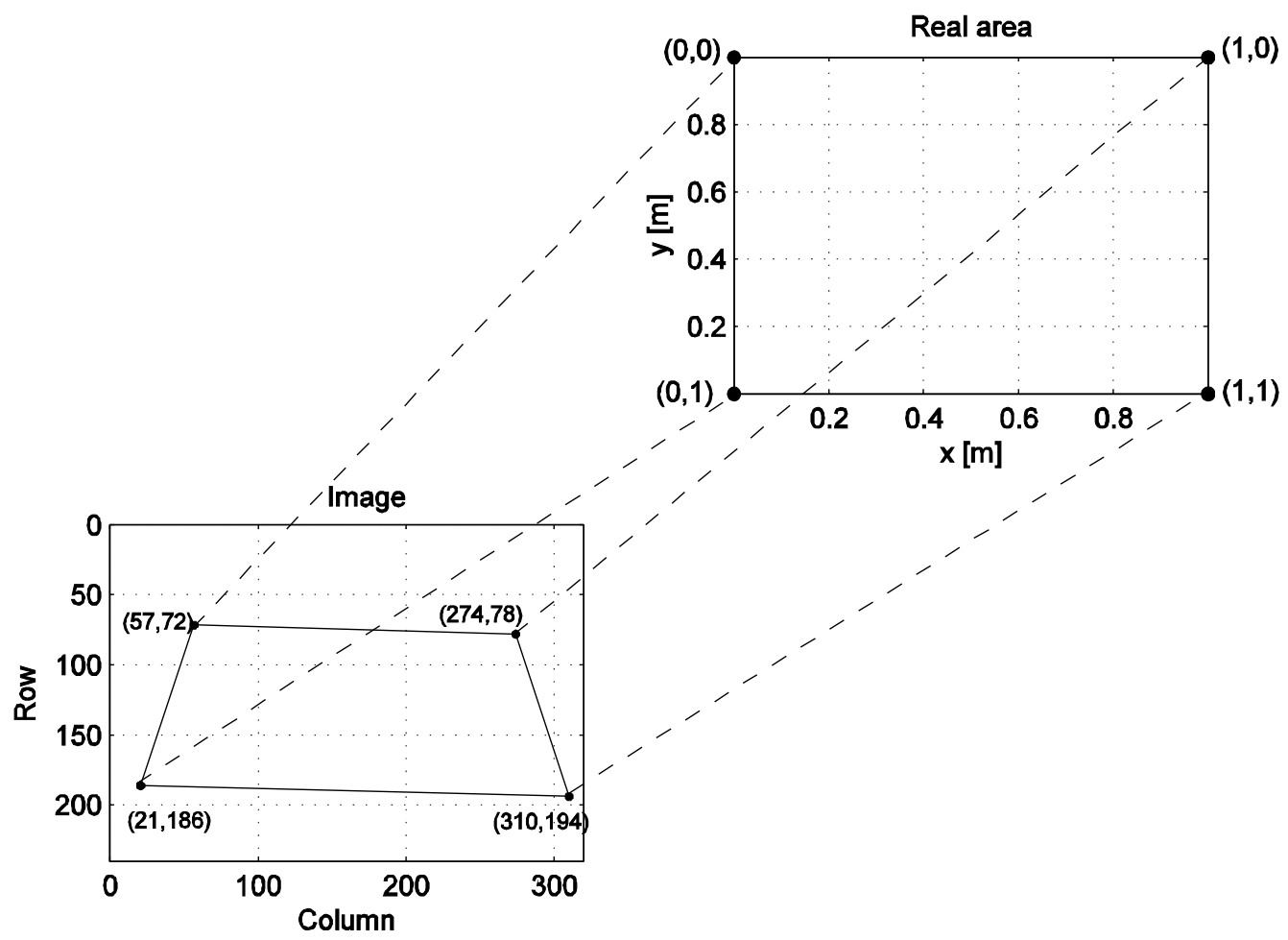


Figure 2.

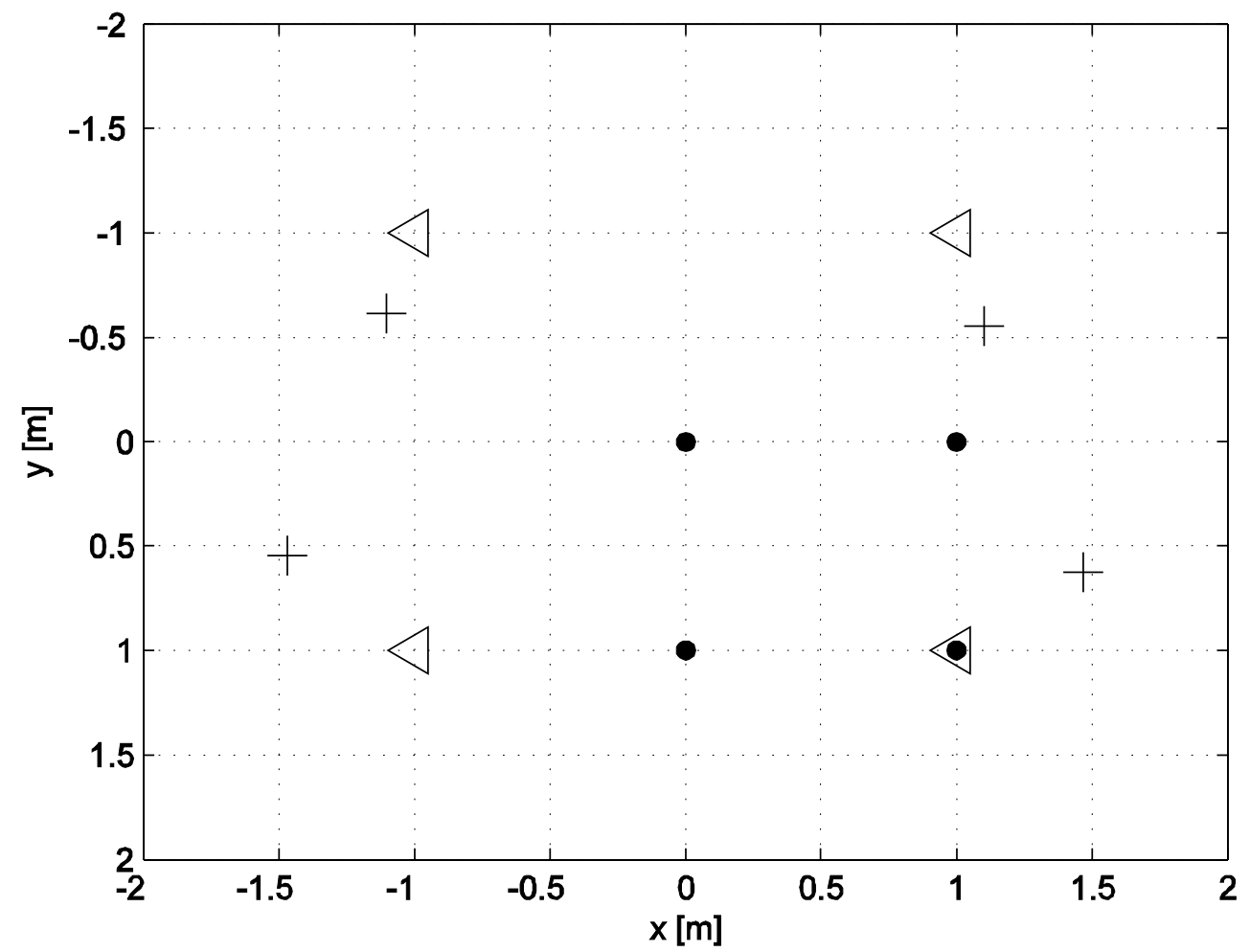

Figure 3. 


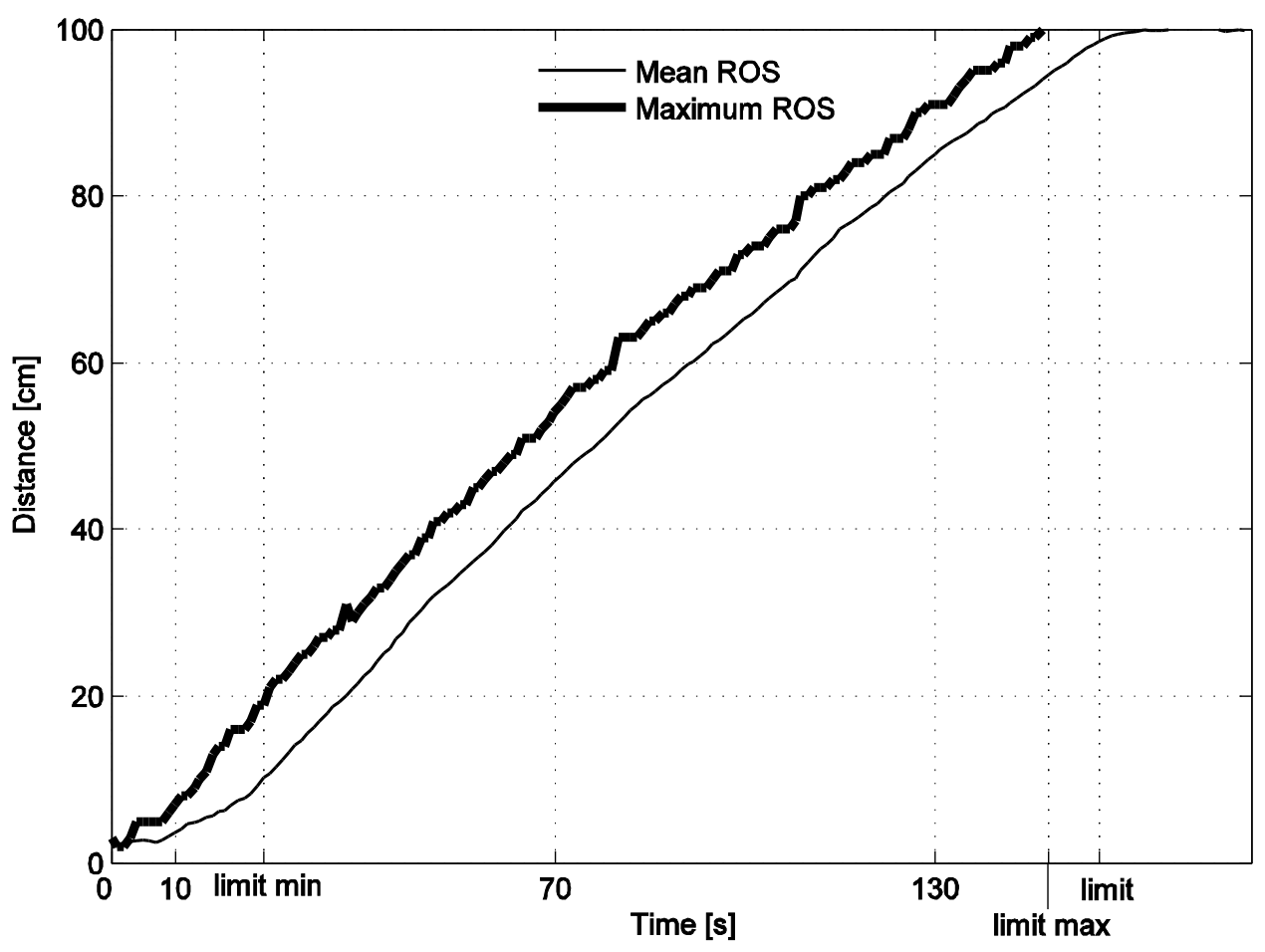

Figure 4a. 


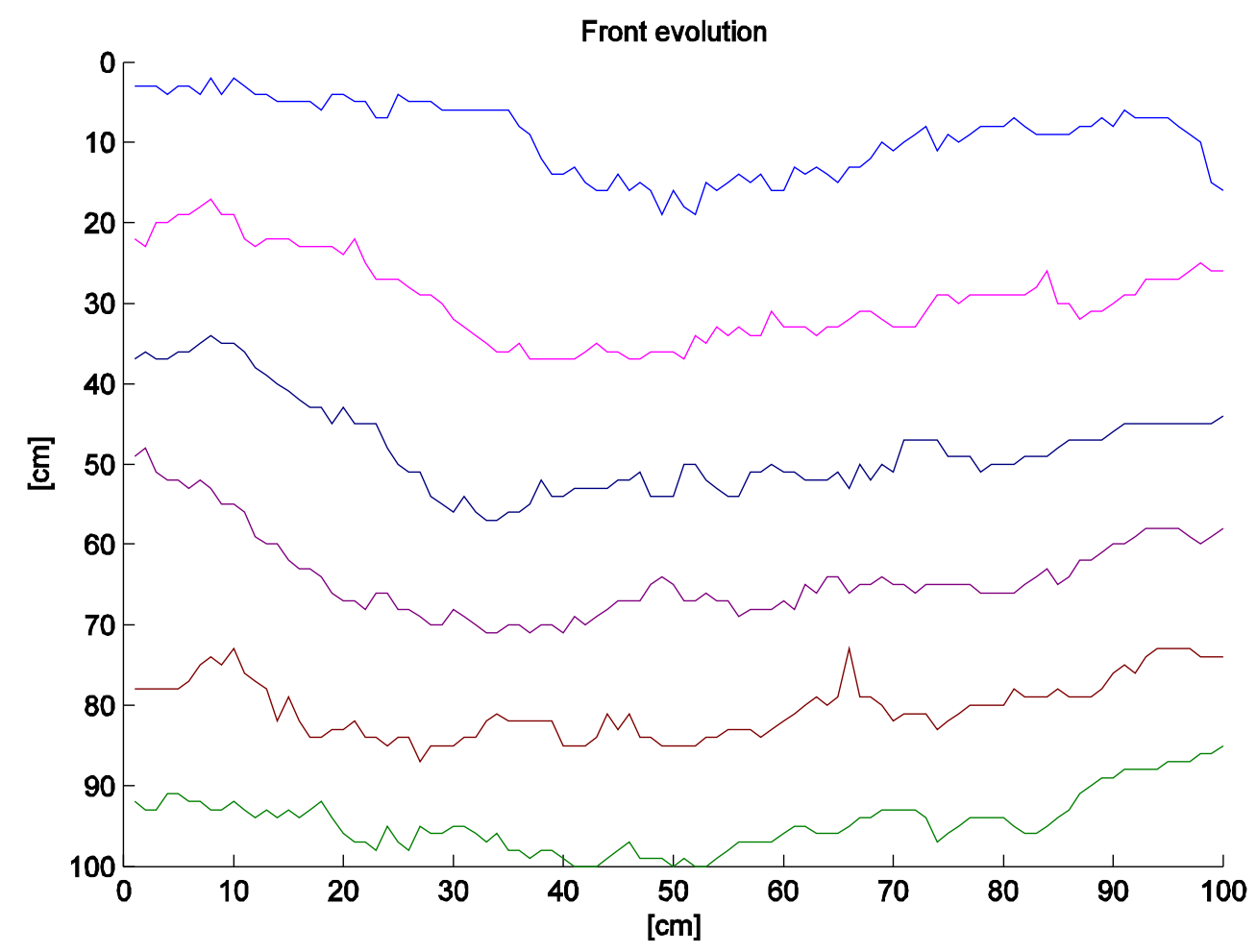

Figure 4b.

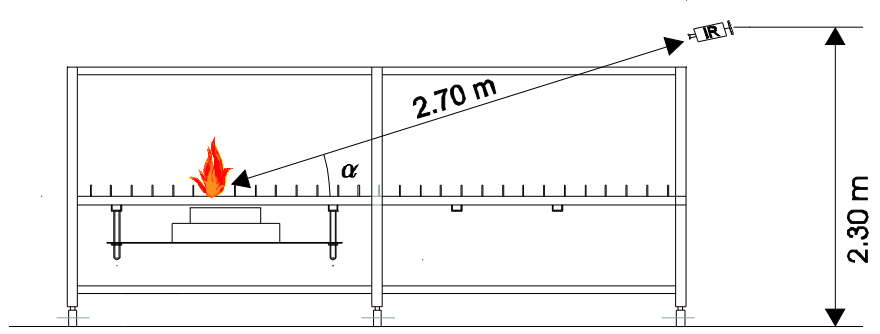

Figure 5. a.

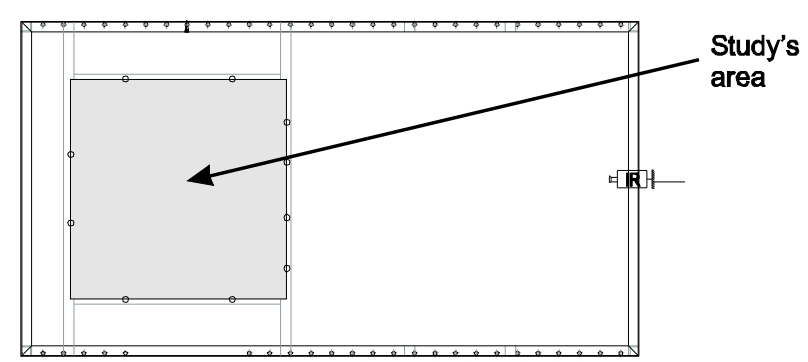

Figure 5b. 


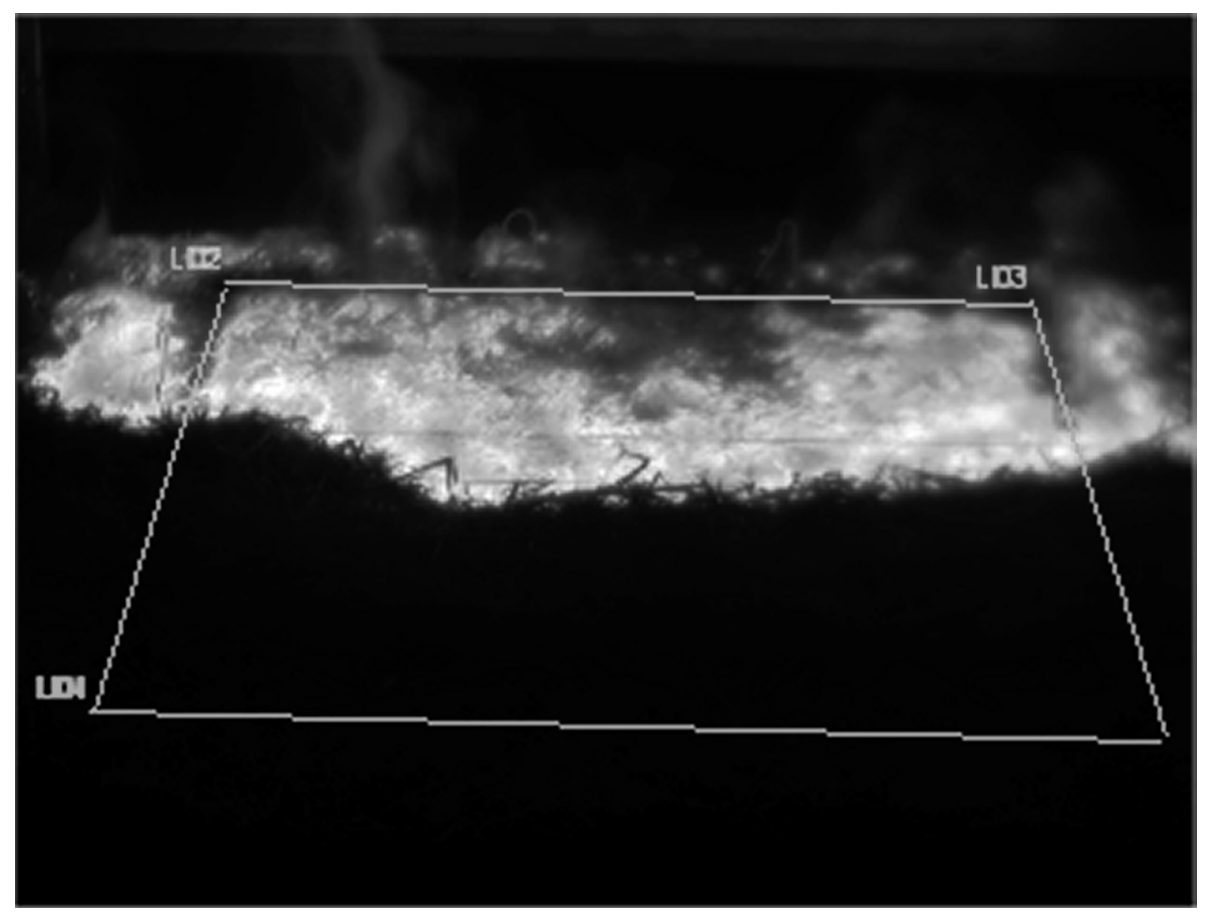

Figure 5c.

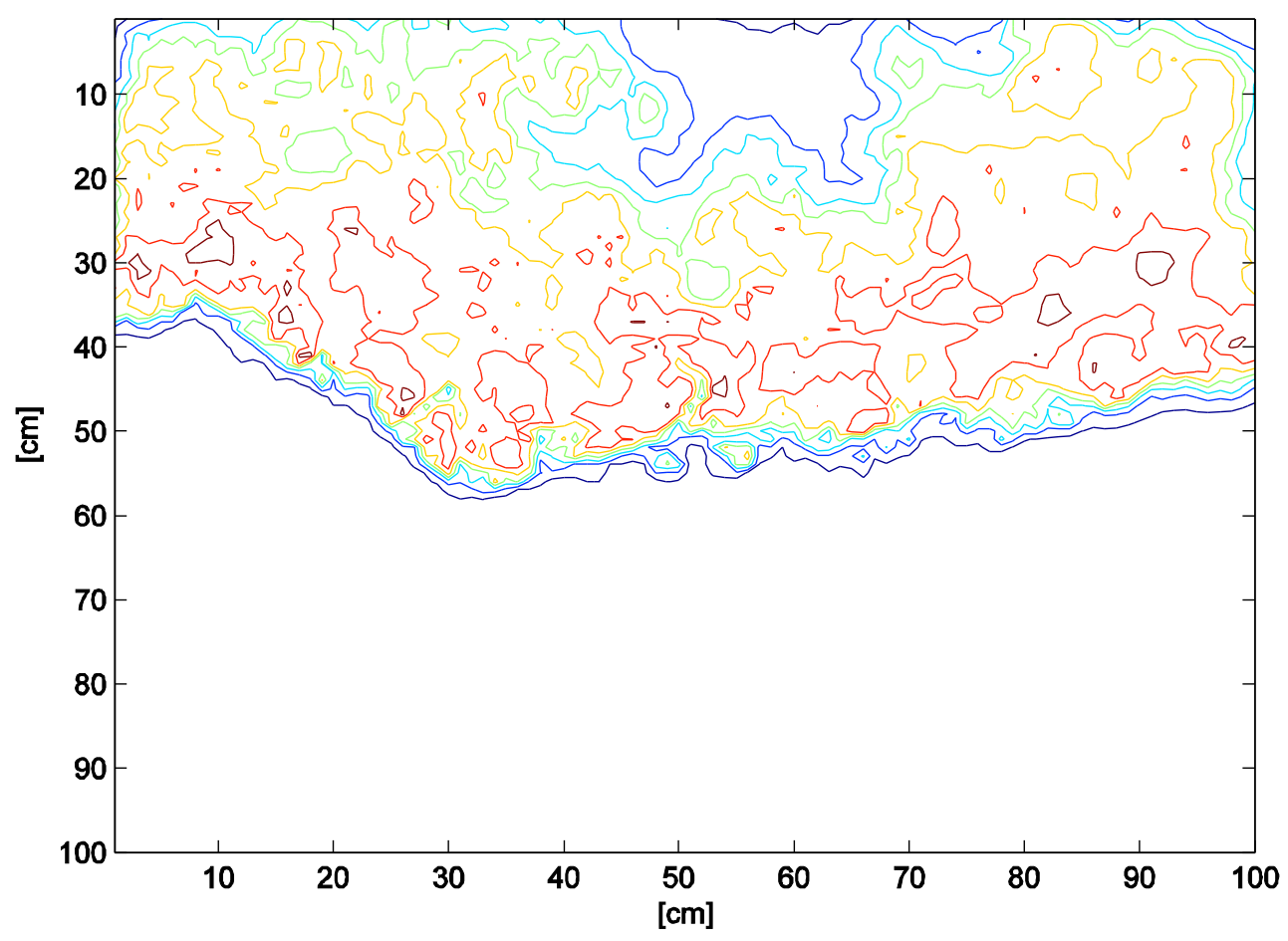

Figure 5d. 


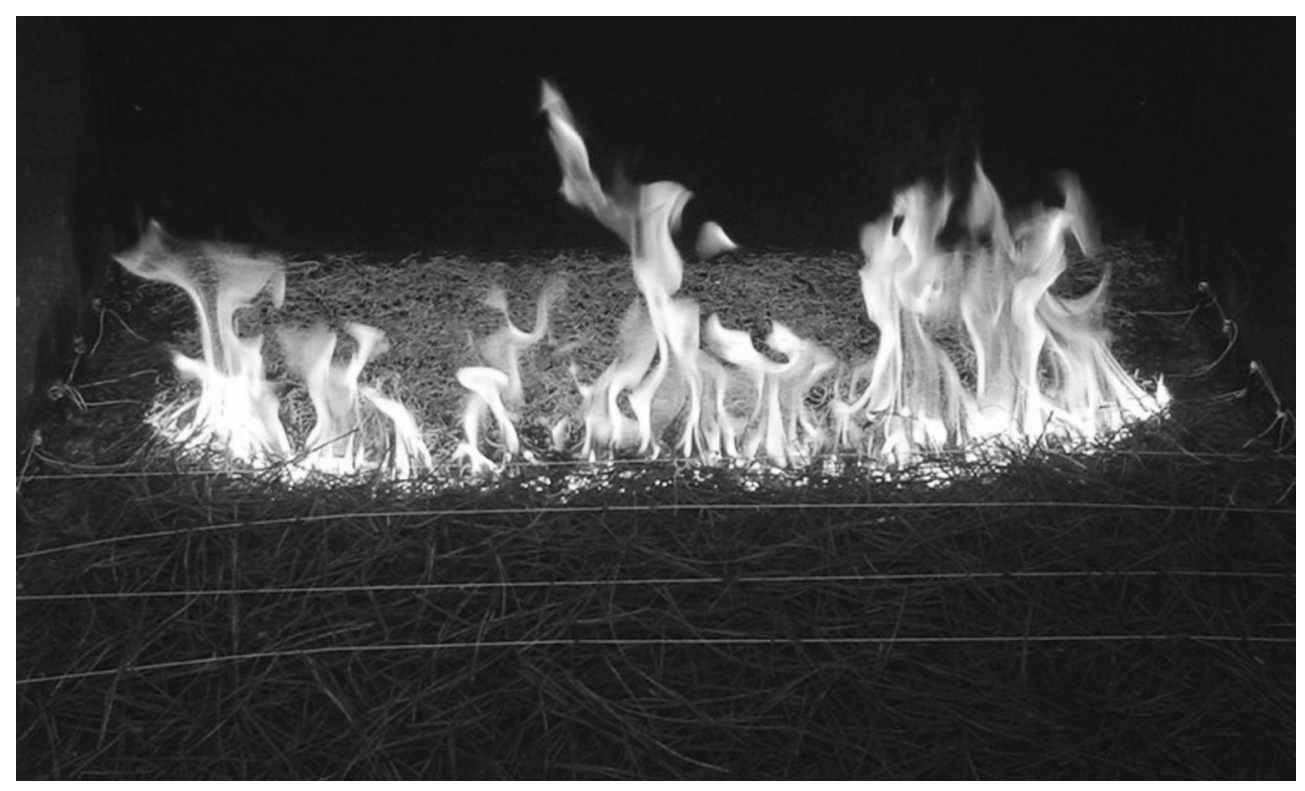

Figure 6.

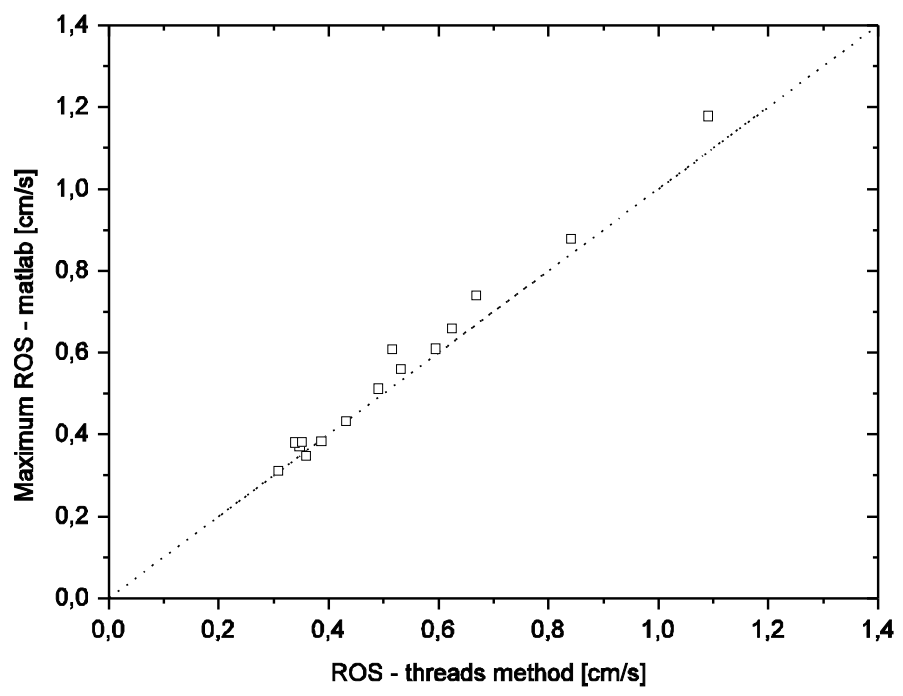

Figure 7. 